\title{
Les journalistes comme scientifiques
}

Notes pour une classification professionnelle

Journalists as Scientists. Notes toward an Occupational Classification

\section{Elihu Katz}

\section{(2) OpenEdition}

\section{Journals}

Édition électronique

URL : http://journals.openedition.org/questionsdecommunication/345

DOI : 10.4000/questionsdecommunication.345

ISSN : 2259-8901

\section{Éditeur}

Presses universitaires de Lorraine

\section{Édition imprimée}

Date de publication : 1 décembre 2009

Pagination : 119-130

ISBN : 978-2-8143-0003-3

ISSN : 1633-5961

\section{Référence électronique}

Elihu Katz, "Les journalistes comme scientifiques », Questions de communication [En ligne], 16 | 2009, mis en ligne le 22 septembre 2015, consulté le 01 mai 2019. URL : http://journals.openedition.org/ questionsdecommunication/345 ; DOI : 10.4000/questionsdecommunication.345

Ce document a été généré automatiquement le 1 mai 2019.

Tous droits réservés 


\title{
Les journalistes comme scientifiques
}

\author{
Notes pour une classification professionnelle \\ Journalists as Scientists. Notes toward an Occupational Classification
}

\section{Elihu Katz}

1 Personne ne sait ce qu'est le journalisme, en tant qu'activité professionnelle ${ }^{1}$. Les journalistes eux-mêmes ne le savent certainement pas. Ils ne se demandent guère s'ils sont une profession, une science appliquée, un art, un divertissement ou une bureaucratie industrielle. Mais parfois, il devient évident que cette question est importante - même pour les journalistes. Quand le quart ou le cinquième des automobiles en Israël porte un autocollant sur le pare-chocs qui proclame : "Le peuple est contre les médias hostiles », ou quand le président de l'État laisse fortement entendre que les journalistes de télévision sont coupables de mauvaises pratiques dans leur couverture du soulèvement palestinien, il est temps de s'interroger sur ce qu'ils considèrent être le journalisme et de pointer ce qui est éventuellement erroné dans leur définition.

Il y a eu plusieurs tentatives de faire entrer le journalisme dans le modèle des professions libérales. En effet, il répond à quelques-uns de ses critères majeurs. C'est avant tout un métier fondé sur la prééminence du service au public, bien que la majorité des journalistes travaillent dans des entreprises de presse privées. L'étude des raisons avancées par les individus pour choisir une profession libérale (Johnstone, Slawski, Bowman, 1976) montre clairement que le désir de participer à la société est une motivation essentielle. Rendre service au client est une caractéristique majeure de cette profession (Blau, Scott, 1963). L'autorité du journalisme, comme les autres professions, est ancrée dans la rationalité plutôt que dans la tradition ou le charisme (Parson, 1968).

Plus encore, le journalisme peut être considéré comme une activité qui se développe à partir du "désordre », comme c'est le cas dans d'autres professions libérales. Everett Hugues (1964) a défini ces dernières comme étant composée de spécialistes qui «fabriquent de routines à partir des urgences des autres». Dans son étude des conversations à l'intérieur des rédactions, Gaye Tuchman (1973) a montré comment les 
journalistes conduisent ce travail de routinisation. Cette recherche des troubles implique la réalisation de diagnostics, bien sûr, et le journalisme parvient également à ce résultat.

4 Les journalistes partagent aussi la norme de la communication privilégiée. Les personnes qui parlent de façon confidentielle à des journalistes peuvent s'attendre à ce que cette confidentialité soit respectée aussi bien par les journalistes que par les tribunaux. Il est aussi considéré comme opportun que les plaintes contre les journalistes soient gérées par des institutions désignées par les journalistes eux-mêmes. Il y a un code professionnel d'éthique, implicite ou explicite, qui guide le travail. Par conséquent, il est évident que, d'une certaine manière, le journalisme est une profession autonome.

5 Mais adopter ce modèle des professions libérales n'est pas sans poser problème. Les médecins et les juristes ont des clients, tandis que les journalistes ne peuvent dire qu'ils en ont qu'en étendant cette catégorie à l'ensemble de la société. Les membres du clergé et les enseignants ont également pour clients l'ensemble de la société, mais leur autorité est fortement tempérée par la tradition ou le charisme. À la différence des autres professions, les clients du journalisme se présentent rarement devant le journaliste ou dans les locaux du journal pour recevoir une aide personnelle. Il est vrai que le journalisme satisfait un large éventail de besoins, mais ce processus ne commence pas avec la présentation de symptômes, une demande de diagnostic, et encore moins un traitement. On peut émettre l'idée selon laquelle les journalistes proposent un traitement, seulement dans le sens où la confrontation avec des informations - et parfois des informations « hostiles » - peut être considérée comme thérapeutique en soi. Après tout, la psychanalyse croit également en la vertu thérapeutique de la confrontation d'informations.

6 Une autre différence consiste en ce que le journalisme rend l'information publique. Comme dans le cas des professions libérales, les professionnels peuvent recevoir des informations privilégiées, mais leur vraie vocation est de les publier. «Publier, et être damné », disait Horace Greeley, indépendamment des conséquences. L'idée que cela puisse n'être que bénéfique est profondément enracinée dans l'idéal libertaire de la démocratie. La façon dont le journalisme s'organise l'éloigne également du modèle professionnel des médecins ou juristes. Enfin, les professions libérales s'adossent à un important volume de connaissances accumulées et à une période intensive de formation qui fait encore défaut au journalisme d'aujourd'hui. Pour toutes ces raisons, le journalisme n'est pas assimilable au modèle des professions libérales. Entre-temps, d'importantes transformations se sont produites au sein de la sociologie des professions libérales. D'une part, la définition du professionnel libéral a été modifiée et s'est en quelque sorte « libéralisé ». D'autre part, l'idéal des professions libérales s'est dégradé si bien que si le journalisme peut être qualifié de profession libérale selon le premier critère, il peut désirer refuser de l'être à partir du second. En effet, les professions libérales sont sujettes aux mouvements induits par les transformations des organisations. L'idéal du professionnel solitaire - avec tous ses droits à un jugement indépendant dans les règles de l'éthique et dans les limites de ses connaissances - s'est maintenant bureaucratisé dans les hôpitaux, les cabinets d'avocats, etc., sans parler des organismes de travail social, des écoles et les institutions qui ont toujours été bureaucratisées.

7 Le savoir des professions libérales a également été déclassé, alors que des analyses distinguent les connaissances véritables des mystifications que les professionnels utilisent pour se protéger des clients et de ceux qui pourraient avoir une mauvaise opinion d'eux. Aujourd'hui, de grands combats sont menés contre les petits caractères et 
les mots imprononçables dans les contrats légaux ou contre le latin des médecins. Ils sont considérés comme autant de conspirations contre le client et le public. S'il reste un peu de sympathie pour ces sortes d'argots, c'est seulement dans la mesure où ils pourraient effectivement servir de raccourcis dans la communication professionnelle.

L'actuel développement des poursuites judiciaires pour faute professionnelle est lié à la mystification du langage et à l'affirmation péremptoire d'une autorité autonome. Le public refuse d'accepter l'autorité des professionnels ou le désintéressement de ses pairs pour décider de ce qui est, ou non, adéquat et fondé sur un plan éthique. Tout compte fait, l'altruisme des professions libérales est continuellement remis en cause et cela remise au rayon de la nostalgie l'image du médecin dévoué jusqu'au sacrifice. Si les journalistes se qualifient en tant que membres d'une profession libérale, ils se trouvent maintenant dans la meilleure - ou devrait-on dire la pire ? - des compagnies. Les professions libérales évoluent dans le sens du journalisme. L'exclusivité de leur savoir est mise en question, leurs compétences placées sous surveillance, la primauté de leur dévouement et de leur altruiste questionnée, leur autonomie individuelle compromise par l'autorité bureaucratique. Il apparaît que « les gens sont également hostiles envers les professions libérales", mais ils sont aussi conscients que, pour l'instant, n'y a pas d'alternative disponible aux médecins et aux avocats. Les journalistes ont une part de responsabilité dans ce processus de dégradation des professions libérales. Peut-être même que certains d'entre eux y trouvent quelque réconfort.

9 Mais la comparaison est loin d'être satisfaisante. Il demeure que le journalisme n'a pas un corpus de connaissances sur lequel il peut s'appuyer ou, si c'est le cas, il n'en est pas explicitement conscient. De même, il n'a pas de procédures réglementaires, et, d'ailleurs, les normes démocratiques rendent celles-ci indésirables. Le journalisme n'a pas de client identifiable, à part peut-être la société dans son ensemble comme cela a été dit. Son but est la publicisation, pas le secret.

\section{I} variante de celui-ci est le modèle des sciences appliquées. Aussi étrange que cela puisse paraitre, je voudrais suggérer que le journalisme répond peut-être plutôt mieux au modèle des sciences appliquée qu'à celui des professions libérales. À première vue, cela semble étrange, non seulement à cause de l'évident manque de cohérence chez les journalistes d'un ensemble de connaissances exigé par la science, mais aussi du fait de l'absence d'un ensemble organisé de propositions - une théorie - et de l'absence de moyens de vérifier ou de tester les implications de la théorie. Néanmoins, testons ce rapprochement.

11 En suivant Edward J. Epstein (1975), considérons le journaliste comme un concepteur de cartes géographiques. Le monde est divisé en zones où des événements importants peuvent survenir et requérir l'attention. Parfois, ces espaces géographiques sont des lieux réels comme Washington, New York ou Jérusalem. Parfois, ce sont des institutions : le ministère des Affaires étrangères, l'armée, la police, les universités et les hôpitaux. Chaque espace comprend une population d'acteurs qui sont détenteurs de pouvoirs légitimes ou non - et dont la plupart sont spécialisés dans la gestion de conflit. Ainsi les informations quotidiennes consistent-elles à donner des indications sur les changements de situation dans ces foyers de pouvoir, habituellement représentées en termes 
d'événements et accompagnée d'évaluations sur les raisons de ces changements de situation, sur qui ou quoi les a provoqués, qui en est affecté et s'il correspond à des normes socialement acceptées (Alexander, 1982). De temps en temps, les journalistes sont contraints de refaire la carte elle-même quand de nouveaux lieux et conflits attirent l'attention.

En d'autres termes, le journalisme a d'abord une fonction de mise à l'agenda en repérant ce qui vaut la peine d'être observé, où et quand. Le journalisme est aussi un baromètre qui indique les changements, habituellement définis comme des événements irruptifs parfois positifs (une percée scientifique dans le domaine médical), mais la plupart du temps négatifs (émeute, mort, famine, impôt...). De façon moins apparente mais tout aussi importante, le rôle du journalisme est de définir et de labelliser les déviances. En attirant l'attention sur les entorses aux normes, il oblige les acteurs politiques à y répondre, soit en punissant la déviance, soit en changeant la norme (Alexander, 1982). Le journalisme donne également la parole aux autres aspects d'un conflit, accordant parfois un « temps égal» aux porte-parole officiels et aux minorités dissidentes. C'est dans cette relation entre conflit et déviation que le journalisme peut être impliqué dans le changement social.

Une assez bonne définition du journalisme est de le comparer à quelque-chose comme la météorologie ; elle parle des entorses à la normalité et des menaces pour le bien-être de la société, ce qu'Harold Lasswell (1948) a appelé la «surveillance». Les météorologues explorent le climat physique; les journalistes explorent le climat social, économique et politique et recherchent les écarts avec le normal ou l'attendu. La société est le client, le diagnostic est la clé d'explication et le traitement n'est généralement pas compliqué. En ce sens, peut-être, le journalisme peut être utilement considéré comme une science, ou, mieux, une science appliquée, comme la météorologie.

Le journalisme ressemble à la science d'une autre façon, via le subtil équilibre entre coopération et compétition parmi les communautés professionnelles. Il y a beaucoup trop, selon certains (voir Noelle-Neumann, 1982) - de coopération parmi les journalistes apparemment en compétition qui partagent des observations, discutent des interprétations et échangent leurs impressions de différentes manières. Il y a également le scoop - la course pour être le premier sur une nouvelle importante - qui pousse les journalistes, comme les scientifiques, à s'empresser de publier, très souvent trop vite, comme l'estiment leurs collègues et les critiques.

Mais quel ensemble de connaissances est mis en œuvre, quelle méthode d'observation est développée, comment sont vérifiées les observations? Nous revenons au lancinant problème de la théorie, des méthodes et de la question de la recherche expérimentale. Il serait absurde d'affirmer que les journalistes disposent d'une théorie abstraite et codifiée, de méthodes sûres et d'une procédure à même de tester les propositions qui dérivent de la théorie. Pourtant, il y a quelque chose à dire à propos du journalisme y compris dans ce domaine.

16 Je pense qu'on peut suggérer que les journalistes avancent des « théories » sur les gens et la société - en fait, chacun de nous en a - mais elles sont latentes, non formulées et non codifiées. Même si les journalistes peuvent être considérés comme envahissants - et je crois qu'ils le sont -, il est néanmoins équitable de se demander quel genre de science cela représente ? Étonnamment, on peut trouver une réponse adéquate en théorie de l'art, et même en physique. Les théories de l'art et de la musique suivent la pratique musicale sur plusieurs années: ce sont des déductions théoriques fondées sur le travail des 
compositeurs et des artistes du champ. Quelque chose de semblable - je le comprends ainsi - peut être dit à propos de la philosophie des sciences qui codifie à nouveau ce que les scientifiques pensent avoir fait. Il est vrai - je suppose - que les compositeurs et les physiciens ont des connaissances et une théorie beaucoup mieux articulés que celles des journalistes. Sans donner raison au journalisme qui ne serait pas respectueux de certaines règles, on ne peut mettre cet aspect de côté. Je ne voudrais pas exclure la possibilité que les journalistes réalisent des déductions théoriques et les soumettent à des tests empiriques. Permettez-moi d'avancer quelques exemples de théories que je pense être implicites à la pratique actuelle du journalisme.

Par exemple, les journalistes peuvent être considérés comme détenteurs d'une théorie volontariste de l'action. À la différence des chercheurs en sciences humaines ou des historiens qui cherchent les contraintes situationnelles et les déterminismes de différentes sortes, ils donnent beaucoup de crédit à l'action humaine autonome. L'idée que des grands hommes, comme Anouar el-Sadate, puissent se retirer dans le désert, prendre une décision et agir pour la mettre en œuvre, est souvent visible dans les journaux, la plupart du temps à une échelle moindre que cet exemple, et c'est valable aussi bien pour les gens malveillants que pour les gens bienveillants. Cependant, nous pourrions faire la distinction - qui, en psychologie sociale, est au centre de la théorie de l'attribution - selon laquelle chaque observateur ordinaire attribue pour les gens bienveillants des motivations "dispositionnelles" à leurs bonnes actions, et des motivations «situationnelles" à leurs mauvaises (l'inverse étant vrai pour les gens malveillants). On peut se demander si les journalistes font cette distinction dans leurs observations. Il serait intéressant de comparer les théories de l'action et de la motivation des journalistes à celles des hommes de loi, par exemple.

Les journalistes croient que le changement social n'est pas seulement l'affaire de personnalités mais d'événements marquants, ce que Victor Turner (1977) appelle les drames sociaux, impliquant l'éclatement de conflits et leur résolution. Quand ils surviennent, les événements sont un anathème pour la plupart des sciences : ce sont des disruptions imprévisibles, alors que la science recherche au contraire la régularité et la prévisibilité. Il y a des événements critiques dans certains domaines, telle la psychanalyse, qui ont été routinisés et régularisés par la théorie. D’autres sciences - la météorologie peut-être - sont plutôt moins préparées à l'inattendu. Mais les journalistes sont également préparés de manière plus ou moins méthodique à l'inattendu. Ils disposent de théories sur les types d'événements et même sur leur périodicité; ils peuvent même prédire la durée de certains genres d'événement (Tuchman, 1973). Ils savent quels événements vont "expulser» les autres de l'agenda public. Il y a une régularité marquée dans l'organisation de la première page d'un quotidien du matin ou dans le conducteur d'un journal télévisé du soir: la guerre, l'émeute, la catastrophe naturelle, la grève, la compétition sportive, le défilé de mode, etc. (Hilgartner, Bosk, 1988). Ceci est aussi une théorie de la société, quels que soient ses précurseurs. Les journalistes ont également une théorie sur la dynamique des événements (voir supra), et ils prédisent ce qui va se passer. Par exemple, je crois que les journalistes "attendent " des troubles civils dans les territoires occupés par Israël, ce qui pourrait expliquer leur attention «exagérée » aux pneus brûlés en Cisjordanie longtemps avant que l'Intifada n'ait commencé.

Bien qu'il soit acquis que les journalistes fabriquent des théories latentes sur les motivations et les actions sociales dans leur façon de construire et de diagnostiquer la 
«réalité », l'analogie quasi-scientifique exige que nous nous posions deux autres questions : 1) Les journalistes contribuent-ils à une accumulation de connaissances dans leur domaine? 2) Ces théories sont-elles susceptibles d'être remises en question et modifiées? Pour la première, je voudrais suggérer que fureter dans les théories latentes des journalistes peut également montrer qu'une telle théorie consiste continuellement à faire face à la dynamique des conflits sociaux. Je ne voudrais pas exclure la possibilité qu'il y ait une certaine accumulation de sagesse non exprimée sur le sujet qui mérite une codification. Mais, parce que la théorie et ses possibles développements sont manifestement peu formulés, on ne peut pas en dire grand chose. Les formations universitaires en journalisme devraient faire de leur mieux pour rendre ces théories latentes manifestes et les confronter aux actuelles théories de la psychologie sociale et de la sociologie. Les journalistes devraient être informés de la théorie de l'attribution ou du débat, chez les sociologues, entre la théorie fonctionnaliste et la théorie du conflit. J'ose suggérer qu'il est également possible qu'un professionnel des sciences sociales ait des choses à apprendre de l'enquête journalistique, du point de vue des éléments de connaissance (à propos des journalistes comme observateurs et des observations des journalistes), mais aussi, autant que possible, de celui des changements de tendance dans la théorie (latente) des journalistes (sur laquelle je suis seulement en train de conjecturer).

\section{II}

Les journalistes ont-ils quelque chose qui puisse être appelé une méthode? Apparemment, une fois encore, la réponse doit être négative. On ne peut dire des journalistes qu'ils ont un esprit systématique pour la mise au jour de la vérité. Ils ne disposent pas des outils de diagnostic du médecin, des outils théoriques de l'homme de loi et certainement pas des instruments ni de la méthodologie du scientifique, ni même du chercheur (empirique) en sciences sociales. J'offenserais les sciences humaines qualitatives comme l'anthropologie ou la psychologie clinique si je laissais entendre qu'il y a des similarités. Mais les journalistes pensent des méthodes à même de produire de la vérité. L'entretien est peut-être la première de ces méthodes. Dans le sillage du Watergate, des conditions préalables rigoureuses sont demandées par la profession (et ses précepteurs légaux) avant que des faits fondés sur de simples entretiens soient acceptés comme des évidences. Des journalistes savent également lire les indicateurs économiques et sociaux, et plus l'usage est fait de ce genre de méthodologie, plus leur façon d'écrire le monde s'éloigne des personnalités et des événements pour se porter sur les tendances à long terme, comme c'est le cas dans les rubriques économique ou médicales.

21 Une nouvelle fois, on pourrait penser que les études universitaires de journalisme - tout particulièrement celles dans les écoles professionnelles de communication - devraient présenter au journaliste ce que le chercheur en sciences sociales connaît de l'entretien ou de la psychologie du témoignage ou encore aborder la question des transformations sociales sur la longue durée. Beaucoup de travail a été fait dans ce domaine (e.g. Trope, 1982) et on peut se demander pourquoi il y a si peu de confrontations et d'échanges entre ces méthodes des sciences sociales et les méthodes traditionnelles de recherche de la vérité dans le journalisme. 


\section{III}

J'espère que ces notes - encore inabouties - sur la comparaison entre le journalisme et les professions libérales, d'une part, entre le journalisme et la science, d'autre part, illustrent l'utilité de ce type d'exercice. Cependant, son intérêt n'est pas seulement celui-ci : il suggère plutôt la mise ne place d'un programme de recherche théorique et méthodologique pour l'enseignement universitaire et la recherche en journalisme et communication. La valeur de cette approche peut être illustrée par la référence à la position décourageante et condescendante d'Edward J. Espstein (1975) sur l'incompatibilité essentielle entre journalisme et vérité. Si notre récapitulatif a quelque valeur, il offre une base pour réfuter ce dernier.

Edward Jay Epstein estime que le journalisme est incapable de dire la vérité. Prenez le cas d'une épidémie de grippe, dit ce dernier. À la différence du médecin, le journaliste est incapable de diagnostiquer la maladie par lui-même: il doit se référer au témoignage d'une autre personne. Cela ne serait pas grave, continue-t-il, si le journaliste avait la capacité de recouper ses sources. Mais non, il doit prendre sa source au pied de la lettre, parce qu'il est incapable de poser les bonnes questions de peur de perdre la confiance de son informateur ou parce que l'heure du bouclage le presse. Il ne peut pas non plus contrôler cette information auprès de collègues reporters de peur de manquer un scoop.

4 Ce qu'il y a d'implicite dans la position d'Edward J. Epstein, c'est bien sûr l'idéalisation de la profession et de la science. Les médecins eux aussi sont souvent incompétents pour réaliser des diagnostics sans l'aide de collègues informés; ils sont également souvent autant pressés par le temps et la compétition que les journalistes. En effet, le sens de l'urgence est une des caractéristiques de la culture professionnelle, et le scoop est une des caractéristiques essentielles de la culture scientifique. Si Edward J. Epstein surestime le médecin, il sous-estime le journaliste. Bien sûr, il est vrai que les journalistes ont besoin d'un expert pour dire qu'il a une épidémie de grippe, mais ils sont suffisamment compétents - à la condition d'être sur les lieux - pour voir par eux-mêmes s'il y a une Intifada. Il y a beaucoup de choses que les journalistes peuvent voir par eux-mêmes, même si l'on doit prendre en compte l'inadéquation typique de leur savoir spécialisé.

Même si nous supposions qu'Edward J. Epstein a raison quand il dit que les journalistes ne font que rapporter ce que les sources établies leur communiquent - ou, s'ils sont des journalistes d'investigation, qu'ils diffusent ce que les sources subversives leur disent -, nous ne devrions pas être si prompts à dévaloriser cet aspect de leur travail. Si un homme dans un costume trois-pièces monte sur une estrade et dit: «je déclare la guerre», le travail du journaliste est de rapporter cette déclaration performative, peut-être de commenter le droit de l'orateur à dire cela, et d'en observer les conséquences. En effet, une partie du travail du journaliste est de diffuser ce que les élites disent et d'en faire un commentaire. Edward J. Epstein élude l'idée que la vérité peut résider dans l'attention professionnelle aux déclarations significatives - indépendamment de la vérité de la déclaration elle-même. Il y a des mots et des actions qui constituent des données objectives que les journalistes sont chargées de collecter et de communiquer. La capacité des journalistes à recouper ses sources est également sous-estimée par Edward Jay Epstein. Effectivement, les journalistes sont souvent dépendants de leurs sources, mais la réciproque est également vraie. Ces dernières années, nous avons vu un important regain dans l'examen critique public des sources dans les interviews en direct à la radio et à la 
télévision. Quelle ironique de constater que cet examen critique soit plus courant à propos du journalisme audiovisuel qu'imprimé, peut-être à cause du caractère irrésistible du passage à la télévision.

Edward J. Epstein explique que la validation par consensus et la vérification indépendante qui caractérisent les professions libérales ne sont pas présentes dans le journalisme. De ce fait, comment expliquer que des auteurs comme Elisabeth Noelle-Neumann (1982) se plaignent que les journalistes soient trop liés les uns aux autres dans une communauté interprétative monolithique de telle sorte qu'ils voient tous les choses de la même façon? Il y a trop de partage, affirme-t-elle, remettant ainsi en cause la représentation d'Edward J. Esptein à propos du journaliste isolé et en compétition avec le temps ou ses collègues.

Nous ne devrions pas seulement prendre en compte la validation par consensus, qui est approprié pour les scientifiques en tant qu'individus, mais prendre aussi en compte la validation institutionnelle, qui est appropriée pour les professions libérales. Finalement, ce peut être un hôpital - avec sa division du travail et sa séparation des pouvoir- et un système de confrontation (pas seulement les avocats en tant qu'individus) qui sont capables de trouver la vérité. C'est une organisation (un hôpital ou un laboratoire scientifique) ou un système (une cour de justice) qui approchent la vérité, mieux que ne le font un praticien privé ou un scientifique isolé. La même chose peut être dite du journalisme. Le journal permet de dire la vérité des faits bien plus que le récit individuel ou le journaliste isolé. Revenant au modèle des professions libérales, on pourrait dire qu'ayant la société comme «client collectif», le journal ou le programme d'information est un « praticien collectif».

\section{BIBLIOGRAPHIE}

Alexander J., 1982, « The mass news media in systematic, historical and comparative perspective », pp. 17-52, in: Katz E., Szecsko T., eds, Mass media and social change, London, Sage.

Blau M. P., Scott W. R., 1963, Formal organization: a comparative approach, London, Routledge/Kegan Paul.

Epstein E. J., 1975, Between fact and fiction, New York, Vintage Books.

Hilgartner S., Bosk C.L., 1988, « Rise and fall of social problems », American Journal of Sociology, 94, pp. 53-78.

Hugues E., 1964, Men and their work, Glencoe IL, Free Press.

Johnstone J., Slawski E., Bowman W., 1976, The news people, Chicago, University of Illinois Press.

Lasswell H., 1948, « The structure and fonction of communication society », pp. 37-51, in: Bryson

L., ed, The communication of ideas, New York, Harper.

Noelle-Neumann E., 1982, pp. 137-166, in: Katz E., Szecsko T., eds, Mass media and social change, London, Sage.

Parsons T., 1968, « Professions », The International Encyclopedia of Social Science, 12, pp. 536-546. 
Trope Y., 1982, « Confirmatory and diagnosing strategies in social information gathering », Journal of Personality and Social Psychology, 43, pp. 22-34.

Tuchman G., 1973, « Making news by doing work: Routinizing the unexpected », American Journal of Sociology, 79, pp. 110-131.

Turner V., 1977, The ritual process: structure and anti-structure, New York, Cornell University Press.

\section{NOTES}

1. Ce texte a été publié en 1989 sous le titre "Journalists as Scientists. Notes Toward an Occupationnal Classification» (The Americain Behavioral Scientist, vol.33, 2, Nov./Dec. 1989, pp. 238-246). Traduction de Vincent Goulet avec le concours de Cécile Boré et Hanna Schops.

\section{RÉSUMÉS}

Ces notes tentent de définir la profession journalistique au moyen d'une comparaison avec les professions libérales et les professions scientifiques. Comme les professions libérales, les journalistes ont le sens du service public et s'occupent des désordres vécus par les individus. Cependant, ils n'ont pas de contacts directs avec leurs clients et ne proposent ni diagnostic ni traitement. La comparaison avec les scientifiques (en particulier les sciences appliquées) parait plus pertinente. Les journalistes ont une théorie intuitive du monde social (une théorie volontariste de l'action, avec des "grands hommes » et des événements décisifs), ils connaissent la dynamique des événements et parviennent à anticiper sur l'inattendu. Cependant, bien qu'ils produisent une forme de vérité socialement construite, ils manquent de méthodes scientifiques, éléments que les écoles formant à la communication pourraient mieux leur transmettre.

Through a comparative approach between the model of "profession liberals" (self-employed white collar workers) and the model of scientists, these notes try to define the journalism activity. As liberal professions, journalism is based on the primacy of public service, making routines of people's emergencies, but journalists haven't direct contacts with their clients: they don't make diagnostic or treatment. Journalists share also with professions the norm of privileged communication but publishing (and not the secret) remain their principal objective. The comparison with the model of applied scientists seems to be more convincing. Journalists have an intuitive theory of the social world (i.e. a voluntary theory of action, with great men and discreet events), they can analyze the unexpected and they know the dynamics of events. Like the community of scientists, journalists work with cooperation and competition. In spite of they can produce a sort of truth, journalists lack reliable methods and accumulated theory. The professional schools of communication could help them to appropriate the tools of social sciences. 
INDEX

Mots-clés : journalisme, profession libérale, scientifique, méthodologie, service public, école de journalisme, vérité

Keywords : journalism, professions, scientists, methodology, public service, school of communication, truth

\section{AUTEUR}

\section{ELIHU KATZ}

Université hébraïque de Jérusalem 